\title{
The behavior of a magnetic filament in flow under the influence of an external magnetic field
}

\author{
Daniel Lüsebrink, ${ }^{1, a)}$ Joan J. Cerdà, ${ }^{1}$ Pedro A. Sánchez, ${ }^{2}$ Sofia S. Kantorovich, $\left.{ }^{2, b}\right)$ \\ and Tomás Sintes ${ }^{1}$ \\ ${ }^{1}$ IFISC (UIB-CSIC) Instituto de Física Interdisciplinar y Sistemas Complejos, Campus UIB, 07122 Palma de \\ Mallorca, Spain \\ ${ }^{2}$ Faculty of Physics, Universität Wien, Boltzmanngasse 5, 1090 Wien, Austria
}

(Received 19 May 2016; accepted 26 November 2016; published online 15 December 2016)

\begin{abstract}
We present an extensive numerical study of the behaviour of a filament made of ferromagnetic colloidal particles subjected to the simultaneous action of a fluid flow and a stationary external magnetic field perpendicular to the flow lines. We found that in the presence of a shear flow, the tumbling motion observed at zero field is strongly inhibited when the external magnetic field is applied. The field is able to stabilise the filament with a well defined degree of alignment that depends on the balance between hydrodynamic and magnetic torques. In addition, for a Poiseuille flow, it has been found that the initial position has a long lasting influence on the behaviour of the magnetic filament when the external field is applied. Published by AIP Publishing. [http://dx.doi.org/10.1063/1.4971860]
\end{abstract}

\section{INTRODUCTION}

The formation of chain-like structures made of ferromagnetic colloids has been predicted more than four decades ago. ${ }^{1}$ Since the pioneering work of Tabata $\mathrm{et} \mathrm{al} .{ }^{2}$ and due to advances in experimental techniques, it is possible to synthesise chains of magnetic colloids with different properties. ${ }^{3-13}$ Recently, Benkoski et al. ${ }^{14}$ have reported the visualisation of ferromagnetic nanoparticles organised into one-dimensional chains, and Hill and Pyun ${ }^{15}$ were able to design one-dimensional structures constructed from polymer-coated ferromagnetic cobalt nanoparticles. The formation of these chains has important implications. For instance, it determines the behaviour of magnetic fluids and their applications. ${ }^{16}$ For a recent review on the chains of magnetic colloidal particles and their applications, see the work of Wang et al. ${ }^{17}$ and the references therein.

Current experimental techniques allow the stabilisation of self-assembled chains of magnetic particles by means of polymer crosslinkers. ${ }^{18}$ The result of this stabilisation is a permanent polymer-like magnetic structure with improved properties with respect to the unstabilised chains. The presence of the crosslinkers produces, for instance, a higher resistance to stresses and an increased magnetic response of the chain. ${ }^{19}$ These crosslinked chains, known as magnetic filaments, are the subject of an increasing research interest due to their potential for technological applications. Their solutions can be thought as easier tunable replacements for conventional ferrofluids; ${ }^{16}$ individual magnetic filaments can be used in magnetic memory devices, as chemical and pressure sensors or in medical applications. ${ }^{20,21}$

\footnotetext{
a)Also at Max-Planck-Institute for the Physics of Complex Systems, Nöthnitzer Str. 3801187 Dresden, Germany; Present address: UCB Department of Physics and Astronomy, University of British Columbia, 6224 Agricultural Road Vancouver, B.C. V6T 1Z1, Canada.

b) Also at Ural Federal University, Lenin Ave. 51, 620000 Ekaterinburg, Russia.
}

The current understanding of the physics of magnetic filaments is far from being complete. Most existing experimental and theoretical works have been devoted so far to the study of their mechanical properties ${ }^{22-24}$ or their use as magnetically controlled micro-propellers and swimmers. ${ }^{18,25-27}$ Several studies have addressed the conformational instabilities that might be experienced due to their interactions with the background fluid. For instance, Cēbers ${ }^{28,29}$ reported on the development of a buckling instability in filaments made of superparamagnetic particles. More recently, the fundamental aspects of the equilibrium configurational behaviour of magnetic filaments made of ferromagnetic particles have been studied theoretically by means of coarse-grained computer simulations. ${ }^{30-32}$ A rich scenario of equilibrium configurations ranging from extended to coiled or closed ring-like and helicoidal shapes has been found. More complex arrangements of magnetic filaments have been investigated using the same approach. See, for example, studies on the polymer brush-like system made of these materials. ${ }^{33,34}$

Many of the aforementioned studies on magnetic filaments are based or focused on their hydrodynamic interactions with a background fluid. However, most of such works correspond to bulk systems. Their behaviour in confined geometries, like the microchannels used in microfluidic applications, ${ }^{35}$ remains almost unexplored. Microchannels are flow pipes with a dimension that ranges from the submicron scale to hundreds of microns. Their high surface-to-volume ratio and small volume provide several advantages for technological applications, like a high rate of heat and mass transfer and a high efficiency in terms of energy and amount of materials. Thus, they can be used as efficient heat exchangers and in the transport, separation, and/or mixing of biological materials and chemical samples. Inside the channel, the pressure driven flow of an incompressible fluid is characterised by a parabolic velocity profile across the diameter. This velocity profile is known as Poiseuille flow. In such confined systems, the competition 
between a spatially dependent shear rate and hydrodynamic interactions leads to a completely different behaviour when compared to the bulk systems. Regarding bodies with a flexible filament structure, this effect has been shown, for instance, in numerous works on the dynamics of flexible polymers in microchannels. In particular, many studies have addressed the conformations and migration across the streamlines of the polymers under such conditions. ${ }^{36-42}$ The use of magnetic filaments within microchannels opens up the possibility to control the hydrodynamic interactions in a microfluidic system by means of external magnetic fields. This may represent a novel approach to design, for instance, highly controllable analyte separation devices, like field-flow fractionation systems. ${ }^{43-45}$

It is the purpose of the present paper to investigate, by means of mesoscopic hydrodynamic simulations, the conformational properties and dynamics of a magnetic filament, made of ferromagnetic colloidal particles, when placed into a microchannel and subjected to the simultaneous action of the fluid flow and an external magnetic field perpendicular to the flow lines. We will consider, for the sake of comparison, two types of flows: a Poiseuille flow that displays a local and smoothly varying shear rate with opposite signs in the upper and lower layers and a Couette flow that is characterised by a uniform shear rate between the channel walls. The coupling of the filament with the flow will be modelled with the multiple-particle collision dynamics (MPC) technique. Our results are expected to properly describe the behaviour of these materials in nano and microfluidic devices and could be of great interest in the understanding of the viscoelasticity and magnetorheological properties of such solutions.

The paper is organised as follows: In Section II we describe the numerical model including the description of the magnetic filament, the solvent, and the simulation setup. In Section III the results are discussed, and finally the conclusions are presented in Section IV.

\section{NUMERICAL MODEL}

In this section, we describe how the filament and the fluid are modelled, as well as our simulation setup for the fluid flow and the external magnetic field. The magnetic filament is implemented as a flexible polymer chain formed by monomers with a permanent magnetic dipole moment. The filament is embedded in an explicit mesoscopic solvent. The different components of the simulation model are described below.

\section{A. Filament}

Among the different experimental techniques available to create magnetic filaments, the one pioneered by Dreyfus et $a l .{ }^{18}$ provides the highest control on the flexibility of the filament backbone. In this approach, neighbouring particles in the chain are crosslinked by chemically bonding the ends of the polymer molecules to their surfaces. On the one hand, the link between the particles can bend more or less easily depending on the amount, length, and intrinsic rigidity of the molecular crosslinkers. ${ }^{46}$ On the other hand, the crosslinkers also constrain the relative rotations of the linked particles: in particular, the crosslinkers usually favor the alignment of their magnetic easy axes. For the case of ferromagnetic particles that have a permanent magnetic moment fixed with respect to their solid body structure, this alignment corresponds to a head-totail orientation of their magnetic moments. Besides this, and to our best knowledge, the understanding of the effects of the experimental crosslinkers on the internal degrees of freedom of the filaments and their overall mechanical properties is still only qualitative. For this reason, we choose to model the magnetic filament and, in particular, the effects of the crosslinkers, using a simple phenomenological coarse-grained approach.

The magnetic filament is modelled as a bead-spring chain of $N$ magnetic monomers with diameter $\sigma$, where monomer $i$ has a dipole moment $\vec{\mu}_{i}$ and a position $\vec{r}_{i}$. As an excluded volume interaction, two monomers $i$ and $j$ interact with each other via a truncated Lennard-Jones potential, which induces purely repulsive interactions between the monomers:

$$
U_{L J}\left(r_{i j}\right)= \begin{cases}4 \epsilon\left[\left(\frac{\sigma}{r_{i j}}\right)^{12}-\left(\frac{\sigma}{r_{i j}}\right)^{6}\right]+\epsilon, & r_{i j} \leq r_{c u t} \\ 0, & r_{i j}>r_{c u t}\end{cases}
$$

where $r_{i j}=\left|\vec{r}_{i}-\vec{r}_{j}\right|$ is the distance between the two monomers and the cut off radius is $r_{c u t}=2^{1 / 6} \sigma$.

The magnetic dipolar interactions are determined by

$$
U_{d i p}\left(\vec{r}_{i j}, \vec{u}_{i}, \vec{u}_{j}\right)=\frac{\vec{\mu}_{i} \cdot \vec{\mu}_{j}}{r_{i j}^{3}}-\frac{3\left(\vec{\mu}_{i} \cdot \vec{r}_{i j}\right)\left(\vec{\mu}_{j} \cdot \vec{r}_{i j}\right)}{r_{i j}^{5}},
$$

where $\vec{r}_{i j}=\vec{r}_{i}-\vec{r}_{j}$. The dipolar interactions are calculated by direct summation over all pairs of particles. Although the total number of interactions that have to be evaluated is of the order $\mathcal{O}\left(N^{2}\right)$, it is the fastest way to compute it because of the relatively small number of monomers involved, with the advantage of being exact. The interactions of the dipoles with an external magnetic field are determined by $U_{H}\left(\vec{\mu}_{i}\right)=-\vec{\mu}_{i} \cdot \vec{H}$, where $\vec{H}$ is the magnetic field.

The beads are connected by a harmonic potential that mimics the effects of the crosslinkers attached to the surface of the monomers. The anchor points of the springs correspond to the intersections of the dipole orientation vectors with the monomer surface (see Fig. 1(a)). This potential creates an energy penalty if the dipoles exhibit orientations different from those tangential to the backbone of the chain and has been introduced in Ref. 31. The interaction between the connected

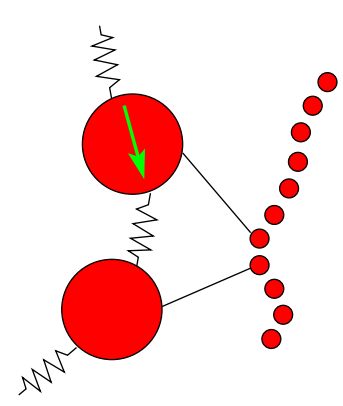

(a)

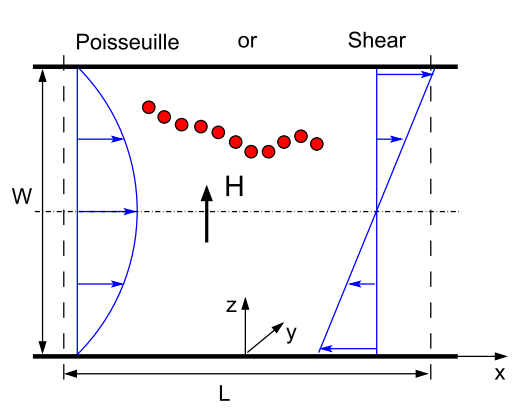

(b)
FIG. 1. Simulation setup. (a) Magnification of the bond arrangements and dipole orientation. (b) Two different flow conditions used in the simulations: Poiseuille and Couette. The external magnetic field acts perpendicular to the flow. 
monomers in the chain is given by

$$
U_{s}\left(\vec{r}_{i j}, \vec{u}_{i}, \vec{u}_{j}\right)=\frac{1}{2} K\left[\vec{r}_{i}-\vec{r}_{j}-\left(\hat{u}_{i}+\hat{u}_{j}\right) \frac{\sigma}{2}\right]^{2},
$$

where $j=i+1, i-1, K$ is the spring constant, and $\hat{u}_{i}=\vec{\mu}_{i} / \mu$ a unit vector director, where $\mu=\left|\vec{\mu}_{i}\right|$ is the magnitude of the dipole moment that is kept constant.

\section{B. Solvent and equations of motion}

For the hydrodynamic coupling of the filament with the flow, we use a mesoscopic method called Multiple-Particle Collision (MPC) dynamics. The method was introduced by Malevanets and $\mathrm{Kapral}^{47}$ and has been successfully used in simulations of complex fluids ranging from colloids or polymers to vesicles, blood cells, active swimmers, and others. ${ }^{48-52}$ Its simplified dynamics, in contrast to other methods like dissipative-particle dynamics, has allowed the analytical calculation of several transport coefficients. MPC can be combined in a hybrid scheme with molecular dynamics (MD) to compute the interactions of the filament which arises from potentials.

In MPC the fluid is represented by explicit point-like particles, which do not correspond to the molecules of the fluid but describe the spatial time evolution of mass, momentum, and energy of the fluid medium. The particle positions $\vec{r}_{i}$ and velocities $\vec{v}_{i}$ are updated in discrete time with alternating streaming and collision steps. In the streaming step, the particles move freely according to

$$
\vec{r}_{i}\left(t+\Delta t_{c}\right)=\vec{r}_{i}(t)+\Delta t_{c} \vec{v}_{i}(t),
$$

with the so-called collision time $\Delta t_{c}$. In the collision step, the particles are sorted into cubic cells of size $a$. In each cell the centre of mass velocity $\vec{v}_{c m}(t)=\sum_{j \mid \vec{r}_{j} \in \text { Cell }} m_{j} \vec{v}_{j}(t) / \sum_{j \mid \vec{r}_{j} \in \text { Cell }} m_{j}$ is computed, where $m_{j}$ is the mass of particle $j$. For all particles in the cell the relative velocities $\Delta \vec{v}_{i}(t)=\vec{v}_{i}(t)-\vec{v}_{c m}(t)$ with respect to the centre of mass velocity are rotated around a random rotation axis by angle $\alpha$,

$$
\vec{v}_{i}\left(t+\Delta t_{c}\right)=\vec{v}_{c m}(t)+R(\alpha) \Delta \vec{v}_{i}(t)
$$

where $R(\alpha)$ is the rotation matrix around the random axis. ${ }^{53}$ The rotation axis is different for each cell and it is randomly chosen in each simulation step whereas the angle $\alpha$ is kept constant. The angle $\alpha$ determines the amount of momentum and energy exchanged in the collisions. For small $\alpha$, the individual particles are less affected by the collision than for large $\alpha$. In order to ensure Galilean invariance, in each collision step, the grid is shifted by a random vector of length $a{ }^{54}$ The average number of particles in one collision cell is $n_{c}$, so that the total number of fluid particles in the simulation box is $N_{f}=n_{c} L^{2} W / a^{3}$, where $W$ is the width of the channel and $L$ the length of the primary simulation box.

The magnetic filament is placed in a three-dimensional slit channel, with planar walls and periodic boundary conditions in the $x-y$ axes in order to mimic an infinite slit (see the scheme in Fig. 1). Fluid particles can penetrate the filament beads and the hydrodynamic interactions between the fluid and the colloids are taken into account by including the chain beads in the collision step $^{55,56}$ and updating their velocities according to Eq. (5). The masses of the fluid particles are $m_{f}$ and the masses of the monomers are set to $m=n_{c} m_{f}$ so that the mass of a monomer equals the average fluid mass in one collision cell. ${ }^{57}$

We have implemented two types of flows: Poiseuille and Couette.

In the case of a Poiseuille flow, we apply a constant acceleration $\vec{g}$ on the fluid particles, which is equivalent to a pressure driven Poiseuille flow and results in a parabolic flow profile. ${ }^{53}$ The flow velocity is related to $\vec{g}$ as $\vec{g}=8 v \vec{v}_{\text {flow }} / W^{2}$, where $v$ is the kinematic viscosity, which is known analytically. ${ }^{58}$ We include virtual particles in partially filled boundary cells to ensure no-slip boundary conditions. ${ }^{53}$

To generate a Couette flow, we move the two walls with the velocity $\vec{v}_{\text {wall }}$ in opposite directions, so that the shear rate is given by $\dot{\gamma}=2 \vec{v}_{\text {wall }} / W$. We implemented stick boundary conditions at the walls via the bounce back rule, where the velocity of a fluid particle $i$ is changed by $\vec{v}_{i}^{\prime}=-\vec{v}_{i}+2 \vec{v}_{w a l l}$, $\vec{v}_{\text {wall }}$ being the velocity of the rigid wall.

In all cases, we apply a thermostat that takes into account the local fluid velocity from which the system temperature, $T_{s}$, is measured. After rescaling all relative velocities by a factor $\sqrt{T / T_{S}}$ the system is kept at a constant average temperature, $T{ }^{48}$ Huang et al. ${ }^{59}$ introduced a thermostat for MPC fluids that takes into account the Poisson distributed particle density in the MPC grid cells and it has been shown to ensure a uniform kinetic energy and density profiles across the slit. With the rescaling thermostat used here, we observe qualitatively similar deviations from the fluid density profiles as in Ref. 59. Such deviations for the range of flow velocities used here are less than $2 \%$ and they do not affect the results regarding the conformations of the filament.

In between MPC steps, the trajectories of the monomers are taken into account by MD, where the number of integration steps is determined by the ratio of the collision time and the MD time step $\Delta t_{c} / \Delta t_{M D}$. For all MD computations of the monomers, we use the ESPResSo package ${ }^{60,61}$ with which we have combined our implementation of MPC.

The equations of motion for the translational and rotational MD integration are, respectively,

$$
\begin{aligned}
m \ddot{\overrightarrow{r_{i}}} & =\vec{F}_{i}, \\
I \dot{\vec{\omega}}_{i} & =\vec{T}_{i},
\end{aligned}
$$

where $\vec{F}_{i}$ is the total force and $\vec{T}_{i}$ is the total torque on a particle due to the interaction among dipoles and the dipoles with the external magnetic field. The hydrodynamic and thermal torques that may be physically important, particularly when acting on a single colloidal particle, are not accounted for in the present study. Even though, the particles are spherical, they are anisotropic due to the presence of the dipole moment in their centres. Thus, the torque applied on the particle in our simulations is equivalent to applying a torque on the vector corresponding to the magnetic moment. $I$ is the angular moment of inertia and $\vec{\omega}_{i}$ its angular velocity.

\section{Simulation details}

The length is measured in units of the MPC grid spacing $r^{*}=r / a$ and time is given in units of $t^{*}=t / t_{0}$ with $t_{0}=\sqrt{m_{f} a^{2} / k_{B} T}$, where the Boltzmann constant is set to $k_{B}$ $=1$. The reduced temperature is $T^{*}=k_{B} T / \epsilon$, the reduced 
dipole moment is determined by $\mu^{*}=\mu / \sqrt{4 \pi \mu_{0} \sigma^{3} \epsilon}$, with the vacuum permeability $\mu_{0}$. The external magnetic field is rescaled as $H^{*}=H \sqrt{4 \pi \mu_{0} \sigma^{3} / \epsilon}$. The magnetic field strength is determined by the Langevin parameter $h=\mu^{*} H^{*} / T^{*}$.

The filament length is set to $N=30$ monomers. Each monomer has a diameter of $\sigma=a$ (with $a=1$ ) and a dipole moment of $\mu^{* 2}=5$. The repulsive interactions have a strength of $\epsilon=1$ and the strength of the springs is set to $K=30$. The parameters for the MPC solvent are $\alpha=120, n_{c}=10$, $\Delta t_{c}=0.1$, and $m_{f}=1$. The resulting kinematic viscosity is $v /\left(k_{B} T a^{2} / m_{f}\right)^{1 / 2} \approx 0.66$ (see Sec. II B). For MD we use a time step of $\Delta t_{M D}=0.0005$, so that the ratio $\Delta t_{c} / \Delta t_{M D}=200$. The width of the channel is $W=50 a$ and the length for the fluid periodic images is $L=75 a$. The solvent temperature is set to $T^{*}=1.5$, since at this temperature chains form open structures, whose behaviour is the scope of the present work. Below a transition temperature of $T^{*} \approx 0.8$, closed structures are formed. ${ }^{31}$ Such different geometries are expected to show a completely different hydrodynamic interaction with the fluid flow and will be the subject of a future study.

Similar to Ref. 41, we characterise the strength of the flow by the Peclet number, which is obtained from $P e=\dot{\gamma} \tau_{0}$. In the case of a uniform shear flow, the shear rate is defined as usual (see Section II B), and for a Poiseuille flow we use a characteristic local shear rate given by $\dot{\gamma}=\partial v(z) /\left.\partial z\right|_{W / 4}=2 v_{\text {flow }} / \mathrm{W}$, similar to a uniform shear flow. $\tau_{0}$ is the relaxation time of the end-to-end vector at equilibrium and has been obtained from the exponential decay of the autocorrelation function. We found $\tau_{0}=3220 t_{0}$, a value that is comparable to other simulations in polymer systems. ${ }^{62}$ In terms of $\tau_{0}$, a MD time step is $\Delta t_{M D}=1.9 \times 10^{-7} \tau_{0}$.

The maximum flow velocity either in the centre for Poiseuille flow or at the walls in the case of a uniform shear is controlled through $v_{\text {flow }}$, such that the Mach number does not exceed $M a=v_{\text {flow }} / v_{s}<0.2$, where $v_{s}=\sqrt{5 / 3 k_{B} T / m_{f}}$ is the sound velocity in an ideal gas, which constitutes the equation of state for the MPC simulation scheme implemented in this paper. In this study, a maximum flow velocity of $v_{\text {flow }}=0.3$ $(P e=31.6)$ is used. Its corresponding Reynolds number is $R_{e}$ $=22.7$ and, although not turbulent, it is significantly beyond the typical $R_{e}<1$ assumed for microfluidics. On addition, the inertia effects due to non-zero and finite $R_{e}$ values will cause some amount of streamline crossing. ${ }^{63}$ Smaller values for $R_{e}$ imply an increase in the fluid viscosity and, as a consequence, larger collision times are needed that require a much higher computational effort. Since the main scope of the work is to evaluate the competition between the flow and the external magnetic field acting on the filament, we can use larger flow velocities combined with higher magnetic fields (up to $h=1.5$ ) with equivalent results.

To integrate the rotational part, for simplicity, we have taken the inertia tensor to be the identity matrix in order to ensure isotropic rotations $I=1$.

The setup of our simulations can be seen in Fig. 1. We use different flow conditions: Poiseuille and Couette, and different maximum flow velocities $v_{\text {flow }}$ at the centre or at the walls, respectively. In Poiseuille flow, we obtain a parabolic flow profile between the two planar walls. In addition, we apply external magnetic fields with varying strength perpendicular to the channel walls along the $z$-direction.

The initial random configuration is let to relax to its equilibrium state. Afterwards, the system is equilibrated until the flow is fully developed, where we use between $3 \times 10^{6}$ and $5 \times 10^{6} \mathrm{MD}$ steps (equivalent to $0.57 \tau_{0}$ and $0.95 \tau_{0}$, respectively). For averaging observables, we have used between $5 \times 10^{6}$ and $2 \times 10^{8}$ MD steps that correspond to the measuring periods of $0.95 \tau_{0}$ and $38 \tau_{0}$, respectively. We have taken 5000 simulation steps between consecutive measurements. The observables in our simulations have been averaged over time and over eight (standard) up to sixteen independent runs. The averages over time and over runs are both denoted by \langle\rangle brackets.

\section{RESULTS AND DISCUSSION}

In this section, we present the results of our simulations and we pay attention to the effect of the magnitude and type of the flow and the strength of the external magnetic field on the behaviour of the magnetic filament.

\section{A. Field inhibited tumbling motion}

In the absence of an applied magnetic field, we observe that the filament under shear undergoes a tumbling motion (shown schematically in Fig. 2). This type of motion has been observed previously in polymers confined in cylindrical micro channels and subjected to Poiseuille flow ${ }^{42}$ as well as in filaments under a uniform shear flow. ${ }^{64}$ The presence of such dynamical effect can be monitored through the total magnetisation of the chain, which is given by $\vec{M}=\sum_{i=1}^{N} \vec{\mu}_{i}$. The total magnetisation is proportional to the end-to-end vector, $\vec{R}_{e e}=\vec{r}_{1}-\vec{r}_{N}$, if the dipoles are aligned along the backbone of the chain, which is the case in our simulations. The change in time of the component of the magnetisation along the channel, $M_{x}$, over its saturation value, $M_{s}=N \mu$, is shown in Fig. 3 for
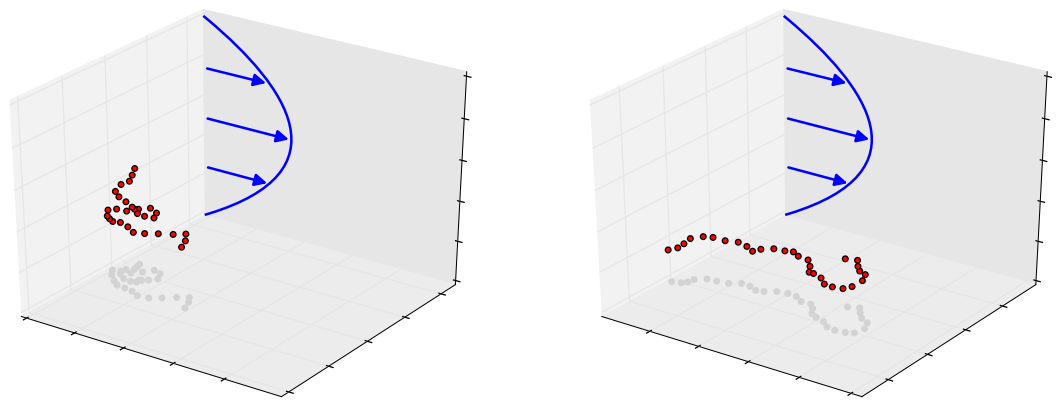

FIG. 2. Two snapshots of the characteristic conformations of a filament subjected to a Poiseuille fluid flow that illustrate the tumbling motion observed at zero field, $h=0$. 


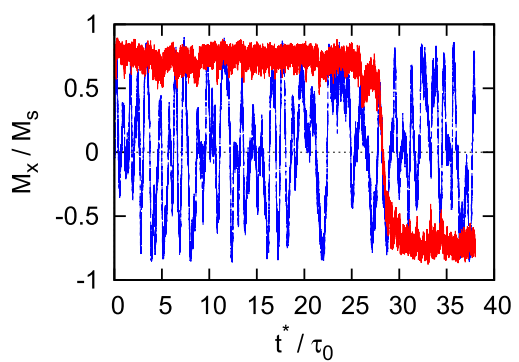

(a)

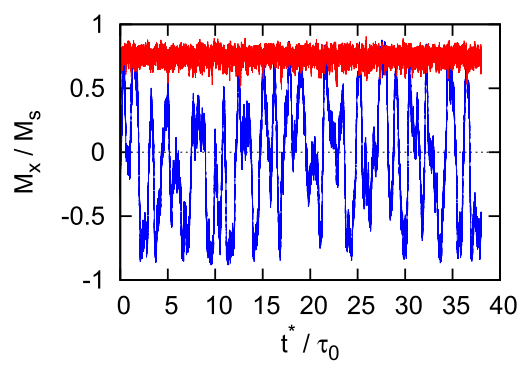

(b)
FIG. 3. Time series of the normalised magnetisation along the flow $M_{x} / M_{s}$. External field: $h=0$ (blue line); $h=1.5$ (red line). Chain length: $N=30$; temperature $T^{*}=1.5$, initial position of the filament: $z=W / 8$; flow velocity $v_{\text {flow }}$ $=0.3(P e=31.6)$. (a) Poiseuille flow. (b) Couette flow. a Poiseuille flow (left) and for a Couette flow (right). We can observe that $M_{x} / M_{s}$ erratically varies between positive and negative values (blue lines), which corresponds to reorientations of the chain in the flow. A steady zero value, $\vec{M}=0$, would correspond to a closed chain, and values near zero are expected for coiled configurations.

In the presence of an external magnetic field perpendicular to the flow, the behaviour of the filament is pronouncedly different. As it can be seen in Fig. 3 (red lines), the magnetisation in the flow direction fluctuates around a constant non zero value. These observations suggest that the tumbling motion with crossovers of the end-to-end vector in the plane perpendicular to the flow is strongly inhibited by the external magnetic field.

For a chain placed in a Couette flow, once the shear flow is coupled with the magnetic field, the alignment of the filament along the flow is retained as depicted in Fig. 3 (right). We checked this behaviour by performing separate long simulations in linear shear flows (data not shown), in which the magnetisation along the flow remained constant for more than 40 times the equilibrium relaxation time of the end-to-end vector.

In the case of a filament placed inside a Poiseuille flow, since the chain is able to slowly diffuse along the $z$-direction, eventually it may cross the centre of the channel where it experiences a change in the sign of the local shear rate. As a result, the filament undergoes fast reorientations until it has entered completely into the new layer. This is reflected in the measure of $M_{x}$ that takes a value that is symmetric with respect to the previous one as seen in Fig. 3 (left). The original position of the $z$-coordinate of the filament centre of mass was $z=W / 8$. It is worth noting that the system is able to retain its orientation beyond $10 \tau_{0}$ for the selected system parameters. Thus, we can define two observation times that are relevant to the Poiseuille flow in our simulations. A short time scale that characterises the average time in which the chain remains in a particular layer with a well defined magnetisation and a long time scale in which the chain is able to diffuse from one layer to another and back. It is worth saying that this division is qualitative: it surely depends on the system parameters and the value of an externally applied field. Besides that, such a distinction is not relevant to the Couette flow. However, splitting the time scales allows us to study the behaviour of the filament in a more systematic and thorough way and deepen our understanding of the relative influence of the initial conditions.

In the supplementary material, we provide videos that illustrate the behaviour of the filament with and without the external field.

\section{B. Chain migration across streamlines}

In an ideal Poiseuille flow, the tendency to push particles far from the walls due to the extra tension in the streamlines in that region is compensated by the effect of the flow to move particles away from the centre of the channel. Such combination of hydrodynamic interactions along the filament and with the walls results in a migration of the chain across the streamlines from the position where it was originally placed until a balance between both effects is achieved.

In order to quantify the relevance of the observed chain migration across the streamlines, we have looked at the evolution of the probability distribution of the $z$ coordinate of the chain centre of mass, $P\left(z_{C M}\right)$ for the case of the Poiseuille flow. The result shown in Fig. 4(a) is obtained after performing long runs, averaged over 16 independent realisations. After a simulation time of $30 \tau_{0}$ (dashed line), a double peak in the distribution emerges. Extending the simulations up to $70 \tau_{0}$ (solid line), a bimodal distribution is obtained in agreement with the

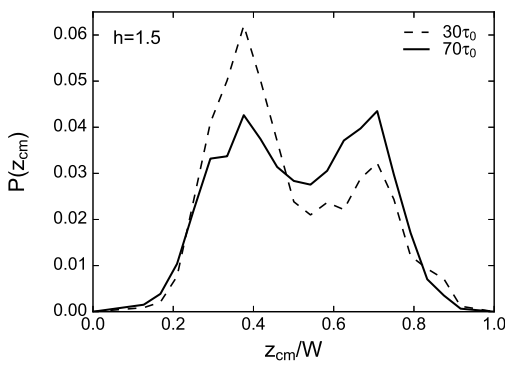

(a)

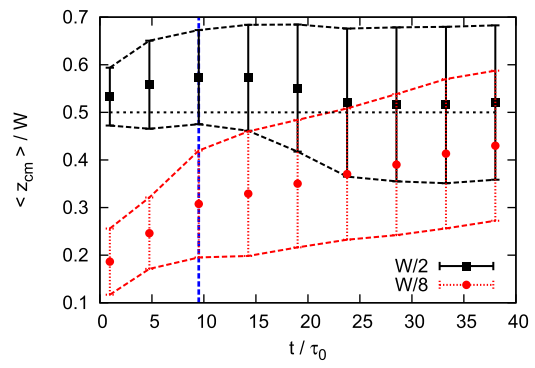

(b)
FIG. 4. Behaviour of the position of the filament centre of mass across the channel, $z_{C M}$, for a Poiseuille flow ( $P e$ $=31.6)$ and an external magnetic field $(h=1.5)$. (a) Probability distributions of $z_{C M}$ for times up to $30 \tau_{0}$ (dashed lines) and $70 \tau_{0}$ (solid lines). Results have been averaged over 16 independent runs. (b) Averaged $z_{C M}$ as a function of time for initial placements at $z=W / 8$ (red dots) and $z$ $=W / 2$ (black squares). The centre of the channel is indicated with the dotted horizontal line. Error bars stand for standard deviation values. 


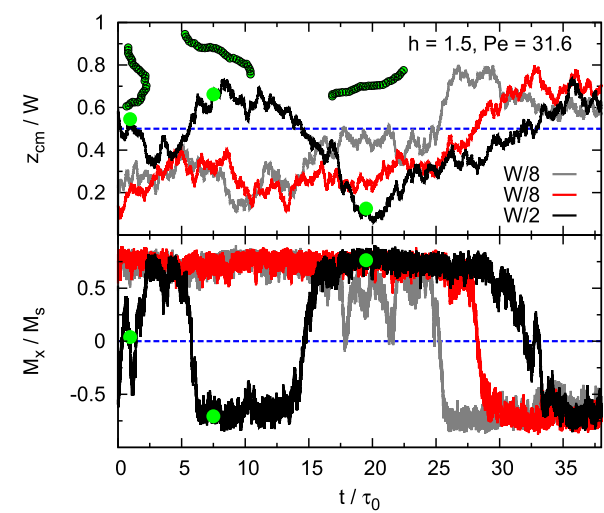

(a)

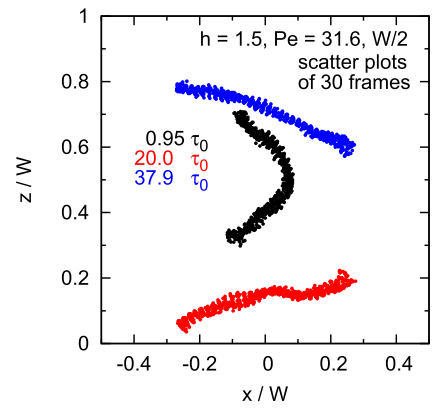

(b)
FIG. 5. (a) Examples of the time evolution of the centre of mass of the filament across the channel, $z_{C M} / W$ (upper panel), and normalised magnetisation along the flow, $M_{x} / M_{s}$ (lower panel), for Poiseuille flow $(P e=31.6)$ and external field $(h=1.5)$. Two trajectories for initial placements at $W / 8$ (grey and red lines) and one for $W / 2$ (black line) are shown. Examples of the three main characteristic filament conformations are included in the upper panel, obtained from the latter trajectory at times indicated by the green points. (b) Overlapped filament conformations corresponding to the locations near the centre of the channel (black) and near the two equilibrium points (red and blue). Each group includes 30 configurations. The overlap is obtained by shifting the $x$-coordinate of the centre of mass of each configuration to zero. findings reported by Reddig and Stark. ${ }^{65}$ The maxima of the distribution located at $z_{C M} / W \approx 0.35$ and $z_{C M} / W \approx 0.7$ should correspond to the equilibrium location of the particles in the channel if no thermal diffusion is present.

The relevance of the initial condition is monitored through the time variation of the average position of the $z$-coordinate of the chain centre of mass, $\left\langle z_{C M}\right\rangle$ up to $t \approx 38 \tau_{0}$ (Fig. 4(b)). In the case of a filament initially placed at $z=W / 8$, the normalised $\left\langle z_{C M}\right\rangle$ has not yet reached its asymptotic value 0.5 (red dotted points), whereas when located at $z=W / 2,\left\langle z_{C M}\right\rangle / W$ basically approaches the centre of the channel (black squared points). The initial condition selects at the beginning the closest equilibrium location, showing its importance on the structural chain properties within the short time scale. Remarkably, the signature of the initial condition has not vanished completely after $t \approx 38 \tau_{0}$.

The existence of two symmetric stable positions within the channel can be better visualised by looking at individual simulation trajectories. In Fig. 5(a) one can see different trajectories of the filament centre of mass (upper panel) and corresponding magnetisation projection on the flow axis for the Poiseuille flow. These examples evidence that the filament fluctuates in the long time scale between the two stable positions, flipping its orientation on the way. The upper panel of Fig. 5(a) also includes examples of the characteristic shapes of the filament at the two stable positions and at the centre of the channel. The persistence of such characteristic conformations is illustrated in Fig. 5(b), where a set of 30 configurations measured for each of the three aforementioned positions has been overlapped.

One can see that at the stable positions the filament is relatively straight, showing a characteristic angle with respect to the direction of the flow, whereas in the middle of the channel the filament assumes the shape of the flow profile, undergoing a flipping transition. The mechanisms governing this behaviour are discussed in detail below.

\section{Filament properties in the short time scale $\left(t \lesssim 10 \tau_{0}\right)$}

In Secs. III C 1 and III C 2 we analyse the degree of alignment of the chain with the flow and its main structural parameters, that is, the radius of gyration and the net magnetic moment projected on the direction of the flow. This will help us to quantify the effect of the strength of the external magnetic field and the fluid flow on the chain behaviour in the short time scale that corresponds to the residence time of the chain under Poiseuille flow in the layer where originally placed.

\section{Chain alignment with the flow}

The degree of alignment of the chain with respect to the flow can be explained by a balance between the hydrodynamic and magnetic torques as shown in Fig. 6 (left). For both Poiseuille and uniform shear flows, the centre of mass of the filament moves with the velocity of the flow, but different parts of the filament are subjected to different velocities due to the imposed velocity gradient. Therefore, the filament experiences an effective torque from the flow rotating clockwise. This hydrodynamic torque is balanced by an effective magnetic torque in the opposite direction due to the external field acting on the dipoles. The net effect of this torque balance is the stabilisation of the alignment of the filament in the flow.

On the right hand side of Fig. 6, we represent the expected orientations of the filament in a Poiseuille flow with respect to the direction of the magnetic field and its position in the channel. The vertical component of the total magnetisation vector upward or downward is determined by the orientation of the external magnetic field. The mainly horizontal orientation of the filament results from the gradient of the flow as has been explained previously. Thus, the filament in a Poiseuille flow can be stabilised at two symmetric positions within the channel, each one with two possible orientations. For the Couette

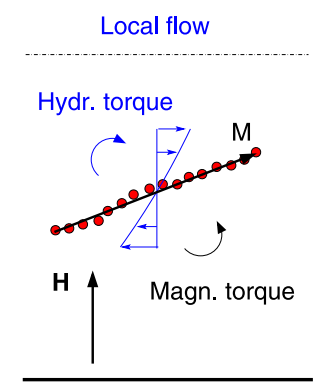

(a)

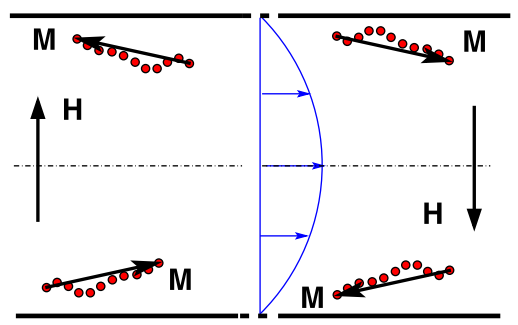

(b)
FIG. 6. (a) Schematic view of the balance between the hydrodynamic and magnetic torques. (b) Possible orientations of the magnetisation of the filament in a Poiseuille flow. 


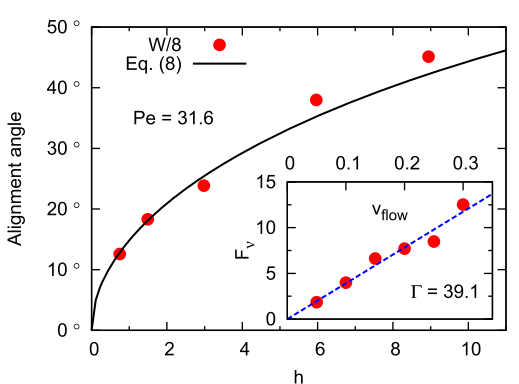

(a)

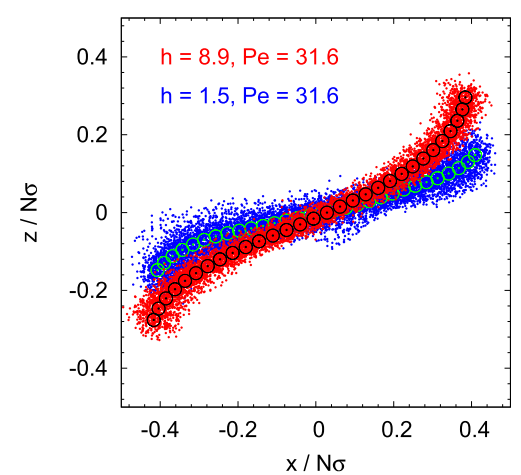

(b)
FIG. 7. (a) Main figure: alignment angle as a function of the magnetic field in a Poiseuille flow. Flow velocity: $v_{f l o w}$ $=0.3(P e=31.6)$. Initial chain location: $W / 8$. Points correspond to simulation data, black solid line to the values given by Eq. (8). Inset shows the fit of the friction coefficient, $\Gamma$, from the simulation data. (b) Scatter plots of the filament conformations observed at positions around $W / 8$ under Poiseuille flow for two values of the external field (red and blue points) and corresponding average conformations (black and green circles). In this visualisation, the centre of mass of each conformation has been shifted to the origin. flow, only one stable position with two orientations can be observed.

In order to analyse the alignment of the filament with the flow at the stable positions, we define the alignment angle, $\theta$, as the angle formed by the end-to-end vector, $\vec{R}_{e e}$, with the $x-y$ plane. In the case of a linear rigid rod, an analytical estimation of this parameter can be obtained by balancing the hydrodynamic and magnetic torques acting on the centre of mass of the filament. Here we give only the basic arguments and the details of the derivation of the exact expressions can be found in the Appendix. The hydrodynamic torque for a filament whose centre of mass is located at $z_{c m}$ and its end-to-end distance is $R_{e e}$ yields an expression that is $\tau_{v} \propto \Gamma \dot{\gamma}\left(z_{c m}\right) R_{e e}^{2} \sin ^{2} \theta$. The torque created by the magnetic field can be written as $\tau_{\mu} \approx N|\vec{\mu} \times \vec{H}|=N \mu H \sin (\pi / 2-\theta)$. By equating these torques, $\tau_{\mu}=\tau_{\nu}$, and assuming that the filament at a stable position has a perfectly straight chain conformation, we obtain an expression that yields $\theta$ as a function of the magnetic interaction, $h=\mu H$, and the flow strength, given by the local shear rate $\dot{\gamma}\left(z_{c m}\right)$,

$$
\Gamma \dot{\gamma}\left(z_{c m}\right) \frac{N^{2}-1}{12} \sigma_{e}^{2} \sin ^{2} \theta=\mu H \cos \theta,
$$

where $\Gamma$ is the friction coefficient of a single bead and $\sigma_{e}$ is the effective distance between consecutive beads in the chain, so that for the straight conformation $R_{e e}=N \sigma_{e}$. Both $\Gamma$ and $\sigma_{e}$ can be directly estimated from the simulation data.

Fig. 7(a) shows the increase of the alignment angle for a filament in a Poiseuille flow, initially placed at $z=W / 8$, with the strength of the magnetic field. The plot includes both the results from the simulation data and the estimation provided by Eq. (8). The latter has been obtained by approximating the local shear rate as corresponding to the average position of the filament centre of mass, $\dot{\gamma}\left(z_{c m}\right) \approx \partial v(z) /\left.\partial z\right|_{z=\left\langle z_{c m}\right\rangle}$, assuming that the variation of $\dot{\gamma}$ along the extension of the filament in $z$ direction is small; in particular, $\left\langle z_{c m}\right\rangle=0.21 \mathrm{~W}$ has been taken; a value of $\Gamma=39.1$, obtained from the linear fit of the hydrodynamic force measured in simulations and the flow velocity (shown in the inset of the figure), has been used; finally, simulation measurements of $R_{e e}$ provided an effective bead diameter of $\sigma_{e}=0.9 \sigma$. The agreement between the simulation data and Eq. (8) is very good, particularly for low values of the field. The higher differences observed at high fields can be explained by the deviations of the filament conformation from a perfectly straight chain. A direct inspection of the filament conformations, illustrated in Fig. 7(b), shows that the filament ends tend to be more aligned with the field than the central part as the field strength increases, making the chain effectively less straight. Therefore, Eq. (8) overestimates the hydrodynamic torque at high fields and predicts smaller angles $\theta$.

The characteristic alignment angle as a function of the strength of the fluid flow is shown in Fig. 8. At zero flow the chain is aligned along the magnetic field, forming an angle of $90^{\circ}$ with respect to the channel walls, as expected. For increasing flow velocities and for a filament subjected to a Poiseuille flow, significant differences are observed depending on the initial position of the filament in the channel. When the chain is placed in the centre (black line) the alignment angle barely changes within the time of observation. This can be easily understood since the chain typically develops a Ushaped structure along the flow due to the different signs in the velocity gradients exerted on different parts of the chain, as shown in Fig. 5(b). Thus, although the magnetisation is small, the alignment is mainly in the direction of the field. On the other hand, if the filament is located outside the centre, for instance at $W / 8$ (red line), it becomes more tilted along the streamlines with increasing flow. At the highest flow velocity the angle is close to $20^{\circ}$. These results are compared with the case of a filament placed at the centre of the channel and subjected to a Couette flow (dashed blue line). The shear rate

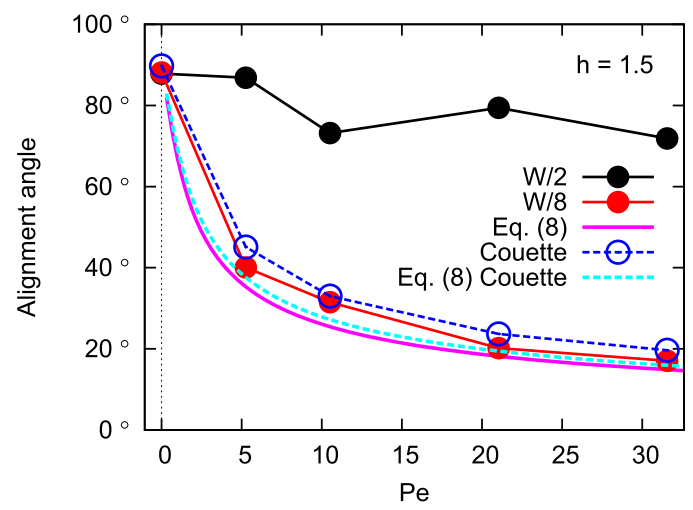

FIG. 8. Alignment angle of the filament as a function of the flow velocity for a strength of the magnetic field $h=1.5$. Filament in a Couette flow placed initially at $W / 2$ (blue dotted lines): simulation results (dotted line with symbols) and corresponding result given by Eq. (8) (dotted line). Filament in a Poiseuille flow (solid lines): simulation results for initial placements at $W / 2$ (black line with symbols) and $W / 8$ (red line with symbols), and corresponding result of Eq. (8) (magenta solid line). 


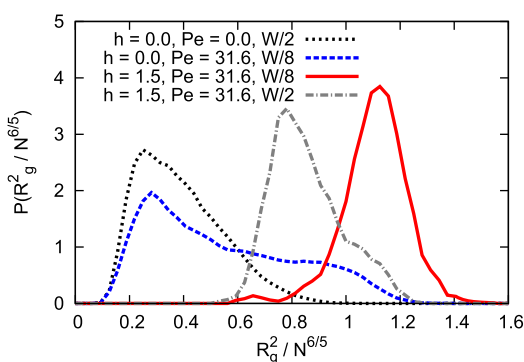

(a)

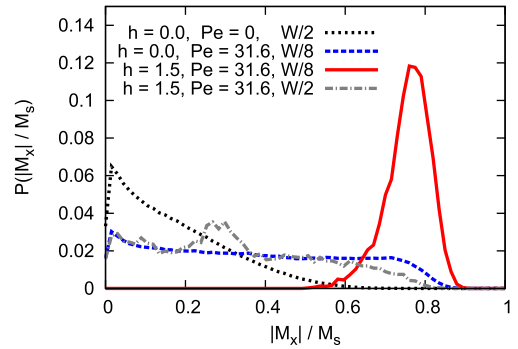

(b)
FIG. 9. Probability distribution of the radius of gyration, $R_{g}$ (a), and the normalised magnetisation along the flow, $M_{x} / M_{s}$ (b), for a filament placed in a Poiseuille flow (Pe $=31.6)$ at different initial positions in the channel evaluated in the short time scale, $t \lesssim 10 \tau_{0}$. At zero field, $h=0$, and $z=W / 8$ (dashed blue line); with an external field $h=1.5$ and $z=W / 8$ (solid red line); at $h=1.5$ and $z=W / 2$ (dotteddashed grey line). For comparison we include the results for a chain in equilibrium: $P e=0, h=0$ (black dotted line). was taken to be equal to the local shear rate in the Poiseuille flow at $W / 8$. The good agreement between the Poiseuille and the uniform shear suggests that the effect of the alignment is induced by the gradient in the flow field and does not occur due to entropic effects of the walls. The agreement of the simulation data with the results of Eq. (8) is also rather good, still showing a slight underestimation of $\theta$ by the analytical approximation.

\section{Structural properties in a Poiseuille flow}

In this section we restrict our analysis to the Poiseuille flow, since significant differences are expected for this profile in the short $\left(t \lesssim 10 \tau_{0}\right)$ and long time scales $\left(t \approx 38 \tau_{0}\right)$. The case of the Couette flow will be analysed when discussing the long time regime.

The locking of the tumbling motion is only one of the effects that the combined presence of a flow and an external field exerts on the behaviour of a magnetic filament. As it will be shown, the flow also strongly modifies the average structural properties of the chains for both zero and non-zero magnetic field cases. In order to characterise such changes, it is convenient to resort to the gyration tensor, defined as

$$
G^{\alpha \beta}=\frac{1}{2 N^{2}} \sum_{i, j=1}^{N}\left(r_{i}^{\alpha}-r_{j}^{\alpha}\right)\left(r_{i}^{\beta}-r_{j}^{\beta}\right),
$$

where $\alpha$ and $\beta$ denote the coordinates $x, y$, and $z$. In the following, we consider the components $G^{\alpha \alpha}$ which represent the elements of the radius of gyration parallel $(x x)$ and perpendicular to the flow ( $y y$ and $z z$ ). $G^{\alpha \alpha}$ are a measure for the average quadratic distance of the monomers from the centre of mass. The radius of gyration can be obtained by the square root of the trace of $G$, so that $R_{g}=\sqrt{\sum_{\alpha} G^{\alpha \alpha}}$ (which corresponds to the sum of the eigenvalues of $G$, since $G$ is quadratic).

As it was already noticed in Sec III C 1, the behaviour of a magnetic filament in a Poiseuille flow is more subtle due to the change in the local shear rate along the perpendicular direction to the flow and the influence of the initial condition. Therefore we focus on this type of flow.

We have evaluated the probability distribution of the radius of gyration and the component of the chain magnetisation in the direction of the flow as a function of the flow intensity and the strength of the external magnetic field. The results are shown in Fig. 9. For a filament in equilibrium at zero flow and zero magnetic field (black dotted line), the probability distributions agree with the earlier findings. ${ }^{32}$ The chain configuration is similar to a random walk, which is slightly more elongated than in the ideal case due to the magnetic dipolar interactions. At the same time, the magnetisation in the $x$ direction exhibits a broad zero centred distribution. In the presence of flow without magnetic field (blue dashed line), the probability distributions of both the radii of gyration and the magnetisation become broader. The reason for wider distribution tails is the presence of tumbling motion induced by the flow. With an external magnetic field and high flow velocities (red solid line), the probability distribution of the radius of gyration is shifted to much higher values, in consistency with the previous results. The magnetisation in this case shows a narrow peak, indicating a persistent orientation of the chain in one direction. The orientations of the dipoles in this case follow the magnetic field but are tilted strongly in the direction of the flow.

The relevance of the initial condition is also reflected in Fig. 9 (dashed-dotted grey line). The radius of gyration for a filament placed at the centre, $W / 2$, shows a well defined peak indicating that the chain is elongated in the direction of the flow. However, it is less stretched than the filament placed at $W / 8$. Similarly, the filament magnetisation when placed at the centre of the channel develops a broad distribution with a non-zero maximum value, but much smaller compared with the filament placed close to the wall, as a result of the fast reorientations of the chain.

The emergence of a well defined peak in the radius of gyration and in the total chain magnetisation with increasing values of the external magnetic field is shown in Fig. 10. At small field values $(h=0.15), R_{g}$ and $\left|M_{x}\right|$ display a broad distribution due to the tumbling motion (black dotted line). At intermediate values $(h=0.45)$, a peak starts to develop (blue dotted line); however, the chain is not yet fully stabilised. At larger field values $(h=1.49)$, the chain is stabilised at a particular alignment angle and a well defined peak is observed (solid red line).

The behaviour of the magnetic filament in a Poiseuille flow can be further analysed through the evaluation of the end-to-end distance $R_{e e}$. In Fig. 11(a) we present the average components of $R_{e e}$ when the chain is subjected to an external magnetic field of varying strength. We observe a monotonic increase of the component along the direction of the field $\left\langle R_{e e, z}\right\rangle$, as expected. More interesting is the behaviour of the component parallel to the flow, $\left\langle R_{e e, x}\right\rangle$. At zero field, its average value is basically zero due to the tumbling motion. As the external field grows, the filament gets stabilised and an abrupt increase in $\left\langle R_{e e, x}\right\rangle$ is observed. However, a further increase in the field strength results in a higher value of the alignment angle, as seen in Fig. 7(a), thus, $\left\langle R_{e e, x}\right\rangle$ tends to 


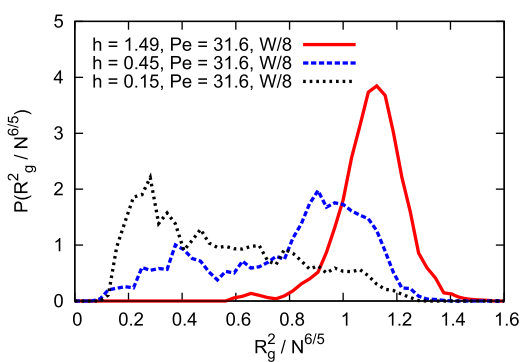

(a)

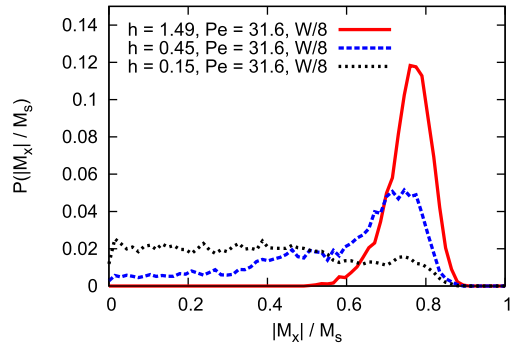

(b)
FIG. 10. Same as Fig. 9, for a chain initially placed at $z$ $=W / 8$ and subjected to a Poiseuille flow of strength $P e$ $=31.6$ at different values of the external magnetic field $h$. decrease for large fields. The interpretation of this behaviour can also be supported by the analysis of the fluctuations of $R_{e e, x}, \Delta R_{e e, x}=\left[\left\langle R_{e e, x}^{2}\right\rangle-\left\langle R_{e e, x}\right\rangle^{2}\right]^{1 / 2}$. Figure 11(b) shows the dependence of $\Delta R_{e e, x}$ on the field strength, in this case for both Poiseuille and Couette flows at $P e=31.6$. The results for both flow profiles are very similar: at low fields $(h \lesssim 0.3)$, large fluctuations of $R_{e e, x}$ can be observed, corresponding to the crossovers of the end-to-end vector in which the filament changes its orientation erratically; at high fields ( $h \gtrsim 1.0)$, the fluctuations are small due to the stabilisation of the main filament orientation. This agrees, respectively, with the broad and sharply peaked distributions observed in Fig. 10 for the magnetisation in the direction of the flow. The intermediate region of field values, $0.3 \lesssim h \lesssim 1.0$, separates the regime of tumbling motion of the filament from the stabilised orientation regime, in which only relatively small thermal fluctuations are present. We note that the transition of thermal to tumbling motion fluctuations occurs at around $h=1$.

\section{Filament properties in the long time scale $\left(t \approx 38 \tau_{0}\right)$}

In Sec III C 2 we have analysed the structural properties of the filament in a Poiseuille flow in the short time scale, a period of time in which the chain remains in the layer where it was originally placed. Such behaviour is expected to be strongly modified in the long time regime due to the diffusion across the channel layers. In addition, we have already observed that the average position of the $z$-coordinate of the chain centre of mass, $\left\langle z_{C M}\right\rangle$, is able to retain the memory of its initial position beyond a simulation time of $t=38 \tau_{0}$ (see Fig. 4(b)). In this section, we will investigate how the influence of the initial condition in a Poiseuille flow is translated into the structural properties in the long time regime. After a simulation time of $38 \tau_{0}$, the response of the filament to the action of the external magnetic field and the flow strength will be analysed through the evaluation of the different components of the radius of gyration. These results will be compared with the case of a filament immersed in a Couette flow that, for simplicity, is treated separately.

\section{Couette flow}

The fastest response of the structural properties of the filament to the flow intensity, with and without the external field, is found to take place when the filament is immersed in a Couette flow, as it is shown in Figures 12 and 13 (blue solid lines). It can be clearly observed from those figures that the sheared flow, characterised here by the Peclet number $P_{e}$, induces on average an elongation of the chain along the flow direction that is independent of the tumbling dynamic phenomenon. Figures 12 and 13 show how the component of the radius of gyration parallel to the flow $G^{x x}$ grows, while the components perpendicular to the flow shrink with $P_{e}$. The net outcome is an increase of the radius of gyration of the chains (notice the star lines in both plots). At this point, it is worth to observe that the radius of gyration at zero flow is in agreement with the earlier findings in Ref. 32.

When the external magnetic field is applied, the tumbling motion is strongly inhibited and the chain remains in a straight configuration. As a consequence, the value of the radius of gyration increases substantially with respect to the zero-field case (see Fig. 13). At zero flow, the filament is elongated mainly in the direction of the magnetic field, whereas with increasing flow the filament bends in the flow direction until the characteristic tilt angle is achieved. It is possible to identify a characteristic flow velocity in which the chain orientation is such that $G^{x x}=G^{z z}$. In an ideal case, and for $G^{y y}$ $=0$, this situation would correspond to a chain tilted at $45^{\circ}$ in the $x-z$ plane.

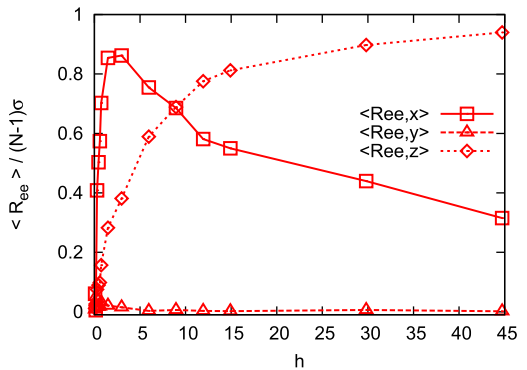

(a)

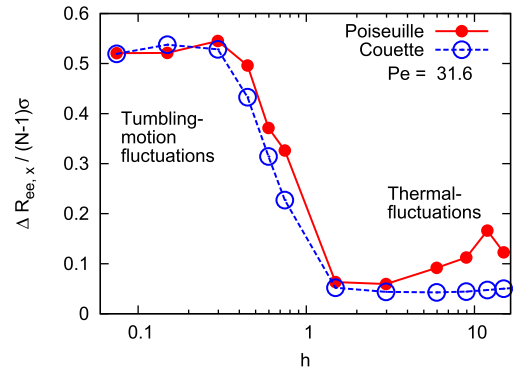

(b)
FIG. 11. Behaviour of the end-to-end distance, $R_{e e}$, of a filament placed initially at $W / 8$ in the short time scale limit ( $t \lesssim 10 \tau_{0}$ ). (a) Average values of the components of $R_{e e}$ normalised by the chain contour length, $(N-1) \sigma$, as a function of the strength of the magnetic field $h$ at $P e=31.6$ for a Poiseuille flow. $\left\langle R_{e e, x}\right\rangle(\square) ;\left\langle R_{e e, y}\right\rangle(\triangle) ;\left\langle R_{e e, z}\right\rangle(\diamond)$. (b) Fluctuations of $R_{e e}, x$ as a function of $h$ for Couette and Poiseuille flows. 


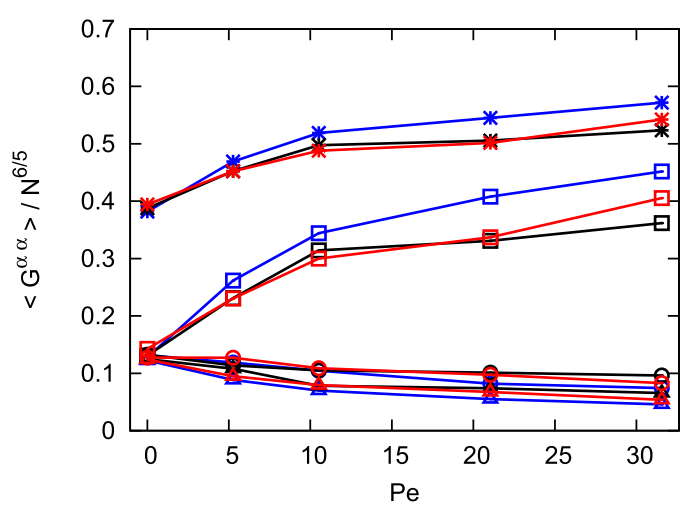

FIG. 12. Filament radius of gyration evaluated in the long time scale regime $\left(t \approx 38 \tau_{0}\right)$ for different strengths of a Poiseuille flow without external field $(h=0)$ and for different initial positions of the filament centre of mass: W/2 (black solid line); $W / 8$ (red solid line). Results when a Couette flow is applied (blue solid line). Symbols stand for: $R_{g}(*) ; G^{x x}(\square) ; G^{y y}(\bigcirc) ; G^{z z}(\triangle)$.

\section{Poiseuille flow}

In the case of a Poiseuille flow, Figures 12 and 13 evidence a behaviour qualitatively comparable to the Couette flow. Moreover, the values obtained in our simulations for the radius of gyration components at zero field are similar to those obtained by Cannavacciuolo et al. ${ }^{41}$ where polymers in narrow channels in Poiseuille flow were studied.

We found the reaction of the chain to the flow to be weaker in the Poiseuille case than in the Couette flow. This effect is due to the fact that in the Poiseuille flow the local shear rate varies with $\mathrm{z}$-position, which induces a local variation of the hydrodynamic torque. Thus, given the same $P_{e}$ number, the effective torque applied over a chain in a Poiseuille flow is smaller than in the case of a uniform shear rate.

We have also analysed the effect of placing the filament at different initial starting positions from the channel centre. In the zero-field case (see Fig. 12), we found almost no differences in the radius of gyration for the different initial positions of the filament. This result is not surprising since the chain dynamics is dominated by the tumbling motion, no matter what its position is in the channel. However, when the external magnetic field is turned on, significant differences can still be appreciated (Fig. 13). The changes in $G^{x x}$ and $G^{z z}$ for a filament initially placed in the centre, $W / 2$ (black solid

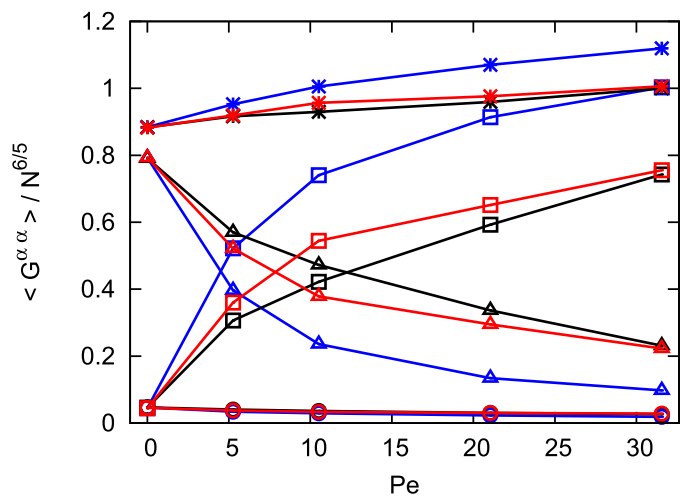

FIG. 13. Same as Fig. 12 when an external magnetic field, $h=1.5$, is applied perpendicular to the fluid flow. lines), are slower compared to the case of a filament located outside the centre, i.e., at the starting position $W / 8$ (red solid line).

Thus, we can conclude that the initial condition has a long lasting influence on the structural properties of the filament in a Poiseuille flow and that significant differences with the Couette flow are observed.

\section{SUMMARY AND CONCLUSIONS}

In this paper we have presented the results of extensive numerical simulations of a magnetic filament made of ferromagnetic particles, placed in a channel and subjected to a fluid flow and an external magnetic field perpendicular to the latter (Fig. 14). We have considered two different flows: Poiseuille and Couette. The simulations combine a hybrid scheme in which fluid particles are coupled to the hydrodynamics via multiple-particle collision dynamics and the interaction between the chain monomers is solved with standard molecular dynamics.

We found that, in the presence of a shear flow, the tumbling motion that is a characteristic of semiflexible chains at zero field is strongly inhibited by the presence of a perpendicular magnetic field. Beyond a characteristic value, the external magnetic field is able to stabilise the filament with a rather straight conformation and a well defined degree of alignment with the flow. The latter is the result of the balance between the hydrodynamic and magnetic torques. Under these conditions, the orientation of the net magnetic moment of the filament is imposed by the direction of the field.

In the case of Poiseuille flow, which is characterised by a parabolic flow profile in $v_{x}(z)$ and a slowly varying local shear rate with opposite signs in the upper and lower layers of the channel, we have been able to identify a short time scale, which depending on the system parameters is typically an order of magnitude larger than the characteristic chain relaxation time (for instance, $t \gtrsim 10 \tau_{0}$, for field intensity $h=1.5$ and flow strength $P_{e}=31.6$ as depicted in Fig. 5(a)), in which the filament tends to remain in the layer where it was originally placed. We have analysed the chain diffusion and migration across the streamlines through the evaluation of the probability distribution of the $z$ coordinate of the chain centre of mass, $P\left(z_{C M}\right)$ and its average position $\left\langle z_{C M}\right\rangle$. We have found

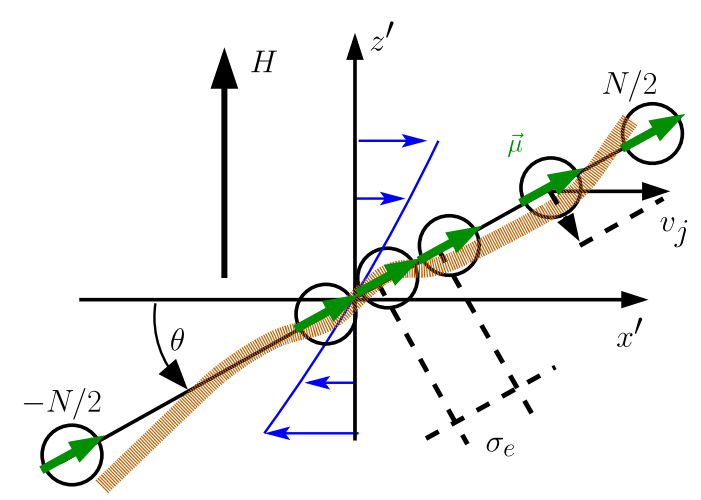

FIG. 14. Schematic figure of a filament immersed into a fluid flow and subjected to an external magnetic field. 
that $P\left(z_{C M}\right)$ develops well defined peaks at a given distance to the channel walls. Such positions, that behave as trapping points, result from the balance between the tendency to push particles far from the walls due to the extra tension in the streamlines and the effect of the flow to move particles away from the centre of the channel. In addition to these effects, thermal fluctuations are responsible for the diffusion across the streamlines.

We have also investigated the relevance of the initial position of the filament across the channel in the case of a Poiseuille flow. In absence of the external magnetic field, the dynamics is dominated by the tumbling motion and there is no evidence of any effect of the initial position. At non-zero field, instead, we found the system to retain the memory of the initial condition over a large period of time. In the short time limit, $t \lesssim 10 \tau_{0}$, the chain diffusion across the streamlines is slow enough so that the filament is strongly influenced by its original position. This is evidenced by the variation of the tilt angle under different flow velocities and the probability distributions of the magnetisation and radius of gyration. For longer times, the filament may reach eventually the opposite layer, where it is forced to adopt an antisymmetric conformation with respect to its stabilised configuration in the former layer. This complete reorientation takes place when the central region is crossed. In the long time limit, $t \approx 38 \tau_{0}$, still clear effects of the initial location are observed in the variation of the radius of gyration with the flow. In this regime, the structural properties of the filament have been analysed and the results for Poiseuille and Couette flows have been compared. We found the Couette flow to provide the fastest response to the flow, as well as the largest values of the radius of gyration.

As a final comment, it is very interesting that a filament stabilised in a Poiseuille flow is able to retain its orientation over a period of time that can exceed, depending on the field intensity and flow velocity, by an order of magnitude its typical relaxation time. This finding can be of great interest in physical processes that take place within this time scale, as it for instance could help to tune the rheological and tribological properties of a fluid containing a finite concentration of those filament units.

\section{SUPPLEMENTARY MATERIAL}

See supplementary material for videos provided to illustrate the tumbling motion of the filament observed at $h=0$ and its stabilisation at $h>0$.

\section{ACKNOWLEDGMENTS}

The authors would like to thank Chien-Cheng Huang, Benjamin Friedrich, Frank Jülicher, and Matthias Merkel for helpful discussions. T.S. and J.J.C. acknowledge financial support from Ministerio de Economa y Competitividad and Fondo Europeo de Desarrollo Regional under Project No. FIS2001563628-C2-2-R (MINECO/FEDER) and a grant awarded by the Conselleria d'Innovaci, Recerca i Turisme del Govern de les Illes Balears and the European Social Fund (ESF). This research has been partially supported by the Austrian Research
Fund (FWF): START-Project No. Y 627-N27. The authors are grateful to the Ural Federal University stimulating programme. S.S.K. is supported by RFBR mol-a-ved No. 15-32-20549. The authors are grateful to the Ministry of Education and Science of the Russian Federation (Contract No. 02.A03.21.000, Project No. 3.12.2014/K) and the Marie-Sklodowska-Curie Project No. H2020-MSCA-ITN-2016, Grant No. 642774, COLLDENSE.

\section{APPENDIX: ALIGNMENT ANGLE IN THE LINEAR ROD APPROXIMATION}

Consider a rigid linear rod built up of monomers with perfectly aligned dipoles. The rod has a length $R_{e e}$ corresponding to the end to end distance of the filament. The angle of the rod, assumed to be oriented in the $x-y$ plane, is given by $\theta$. Then the total magnetic torque can be written as

$$
\tau_{\mu}=N \mu H \cos \theta .
$$

This magnetic torque is counterbalanced by a hydrodynamic torque due to the flow, which can be calculated as follows.

Neglecting perturbations of the flow through the filament particles, each monomer $j$ experiences a local excess flow velocity $v_{j}=v\left(z_{j}\right)-v\left(z_{c m}\right)$ in the co-moving reference frame of the centre of mass of the filament, where $z_{j}$ is the $z$-position of monomer $j, z_{\mathrm{cm}}$ is the centre of mass of the filament, and $v(z)$ is the flow velocity in the channel. We assume that the local flow velocity can be approximated by

$$
v_{j}=z_{j}^{\prime} \dot{\gamma}\left(z_{c m}\right),
$$

where we have introduced the local coordinates $z_{j}^{\prime}=z_{j}-z_{\mathrm{cm}}$. In the case of Couette flow, this is true in any case and $\dot{\gamma}\left(z_{c m}\right)=\dot{\gamma}$. For Poiseuille flow $\dot{\gamma}\left(z_{c m}\right)=\partial v(z) /\left.\partial z\right|_{z=z_{c m}}$ $=4 v_{\text {flow }} / W\left(2 z_{c m} / W-1\right)$, since $v(z)=-v_{\text {flow }}(2 z / W-1)^{2}$ $+v_{\text {flow }}$. The local force that the monomer $j$ experiences due to hydrodynamic friction is given by

$$
F_{j}=\Gamma v_{j},
$$

where $\Gamma$ is the friction coefficient of a monomer (see inset in Fig. 7(a)). In order to calculate the torque around the center of mass of the filament, we introduce the effective distance between monomers $\sigma_{e}$ such that the total length of the rod is given by $R_{e e}=\sigma_{e} N$. The effective distance $\sigma_{e}$ basically accounts for thermal fluctuations and deviations from a linear shape. The distance of a monomer from the center of mass is then given by $d_{j}=\sigma_{e}(j+1 / 2)$, where $j=-N / 2 \ldots N / 2$. Therefore, taking into account the friction force perpendicular to the rod, the torque around the filament center of mass due to monomer $j$ is

$$
\begin{aligned}
\tau_{j} & =F_{j}(j+1 / 2) \sigma_{e} \sin \theta \\
& =\Gamma \dot{\gamma}\left(z_{c m}\right)(j+1 / 2)^{2} \sigma_{e}^{2} \sin ^{2} \theta,
\end{aligned}
$$

where we have used Eqs. (A2) and (A3) and

$$
z_{j}^{\prime}=(j+1 / 2) \sigma_{e} \sin \theta .
$$


The total hydrodynamic torque $\tau_{v}$ is given by summing the contributions of all $N$ monomers,

$$
\begin{aligned}
\tau_{\nu} & =\sum_{j=-N / 2}^{N / 2} \tau_{j} \\
& =\Gamma \dot{\gamma}\left(z_{c m}\right) \sigma_{e}^{2} \sin ^{2} \theta \sum_{j=-N / 2}^{N / 2}(j+1 / 2)^{2} .
\end{aligned}
$$

Using that $\sum_{j=-N / 2}^{N / 2}(j+1 / 2)^{2}=1 / 12\left(N^{3}-N\right)$ we finally obtain

$$
\tau_{v}=\dot{\gamma}\left(z_{c m}\right) \Gamma \frac{N\left(N^{2}-1\right)}{12} \sigma_{e}^{2} \sin ^{2} \theta .
$$

For odd numbers $N$, one can derive the same result with different indexing and a different definition of $z_{j}^{\prime}$. Equating the two torques in Eqs. (A1) and (A9) yields Eq. (8).

Eq. (8) is a quadratic equation in $\cos \theta$ and we have used the positive root for the lines in Fig. 7(a) and Fig. 8.

${ }^{1}$ P. Gennes and P. Pincus, Phys. Kondens. Mater. 11, 189 (1970).

${ }^{2}$ O. Tabata, H. Kojima, T. Kasatani, Y. Isono, and R. Yoshida, "Chemomechanical actuator using self-oscillating gel for artificial cilia," in Proceedings IEEE Sixteenth Annual International Conference on Micro Electro Mechanical Systems (IEEE, 2003), pp. 12-15.

${ }^{3}$ H. Singh, P. E. Laibinis, and T. A. Hatton, Langmuir 21, 11500 (2005).

${ }^{4}$ H. Singh, P. E. Laibinis, and T. A. Hatton, Nano Lett. 5, 2149 (2005).

${ }^{5}$ J. den Toonder et al., Lab Chip 8, 533 (2008).

${ }^{6}$ B. A. Evans et al., Nano Lett. 7, 1428 (2007).

${ }^{7}$ Z. Zhou, G. Liu, and D. Han, ACS Nano 3, 165 (2009).

${ }^{8}$ P. Y. Keng, I. Shim, B. D. Korth, J. F. Douglas, and J. Pyun, ACS Nano 1, 279 (2007).

${ }^{9}$ S. E. Bowles et al., J. Am. Chem. Soc. 129, 8694 (2007).

${ }^{10}$ J. J. Benkoski, H. Hu, and A. Karim, Macromol. Rapid Commun. 27, 1212 (2006).

${ }^{11}$ J. J. Benkoski, R. L. Jones, J. F. Douglas, and A. Karim, Langmuir 23, 3530 (2007).

12 J. J. Benkoski et al., J. Am. Chem. Soc. 129, 6291 (2007).

${ }^{13}$ J. J. Benkoski et al., J. Polym. Sci., Part B: Polym. Phys. 46, 2267 (2008).

${ }^{14}$ J. J. Benkoski et al., Soft Matter 6, 602 (2010).

${ }^{15}$ L. J. Hill and J. Pyun, ACS Appl. Mater. Interfaces 6, 6022 (2014).

${ }^{16} \mathrm{~S}$. Odenbach and S. Thurm, "Magnetoviscous effects in ferrofluids," in Ferrofluids: Magnetically Controllable Fluids and Their Applications, Lecture Notes in Physics, edited by S. Odenbach (Springer, Berlin, Germany, 2002), Vol. 594, pp. 185-201.

${ }^{17}$ H. Wang, Y. Yu, Y. Sun, and Q. Chen, Nano 06, 1 (2011).

${ }^{18}$ R. Dreyfus et al., Nature 437, 862 (2005).

${ }^{19}$ P. A. Sánchez, J. J. Cerdà, T. Sintes, A. O. Ivanov, and S. S. Kantorovich, Soft Matter 11, 2963 (2015).

${ }^{20}$ C. Goubault et al., Phys. Rev. Lett. 91, 260802 (2003).

${ }^{21}$ S. A. Corr et al., J. Am. Chem. Soc. 130, 4214 (2008).

${ }^{22}$ S. L. Biswal and A. P. Gast, Phys. Rev. E 68, 021402 (2003).

${ }^{23}$ K. Ērglis, L. Alberte, and A. Cēbers, Magnetohydrodynamics 44, 223 (2008).

${ }^{24}$ K. Ērglis, D. Zhulenkovs, A. Sharipo, and A. Cēbers, J. Phys.: Condens. Matter 20, 204107 (2008).

${ }^{25}$ A. Cēbers and I. Javaitis, Phys. Rev. E 69, 021404 (2004).

${ }^{26}$ A. Cēbers, Curr. Opin. Colloid Interface Sci. 10, 167 (2005).
${ }^{27}$ M. Belovs and A. Cēbers, Phys. Rev. E 79, 051503 (2009).

${ }^{28}$ A. Cēbers, J. Phys.: Condens. Matter 15, S1335 (2003)

${ }^{29}$ A. Cēbers, J. Magn. Magn. Mater. 300, 67 (2006).

${ }^{30}$ P. A. Sánchez et al., Soft Matter 7, 1809 (2011).

${ }^{31}$ J. J. Cerdà, P. A. Sánchez, C. Holm, and T. Sintes, Soft Matter 9, 7185 (2013)

${ }^{32}$ P. A. Sánchez, J. J. Cerdà, T. Sintes, and C. Holm, J. Chem. Phys. 139, 044904 (2013).

${ }^{33}$ P. A. Sánchez et al., Macromolecules 48, 7658 (2015).

${ }^{34}$ P. A. Sánchez et al., Faraday Discuss. 186, 241 (2016).

${ }^{35}$ N.-T. Nguyen and S. T. Wereley, Fundamentals and Applications of Microfluidics (Artech House, Inc., Norwood, USA, 2002).

${ }^{36}$ R. M. Jendrejack, E. T. Dimalanta, D. C. Schwartz, M. D. Graham, and J. J. de Pablo, Phys. Rev. Lett. 91, 038102 (2003).

${ }^{37}$ R. M. Jendrejack, D. C. Schwartz, J. J. de Pablo, and M. D. Graham, J. Chem. Phys. 120, 2513 (2004)

${ }^{38}$ J. O. Tegenfeldt et al., Proc. Natl. Acad. Sci. U. S. A. 101, 10979 (2004).

${ }^{39}$ D. Stein, F. H. J. van der Heyden, W. J. A. Koopmans, and C. Dekker, Proc. Natl. Acad. Sci. U. S. A. 103, 15853 (2006).

${ }^{40}$ A. Balducci, C.-C. Hsieh, and P. S. Doyle, Phys. Rev. Lett. 99, 238102 (2007).

${ }^{41}$ L. Cannavacciuolo, R. G. Winkler, and G. Gompper, EPL 83, 34007 (2008).

${ }^{42}$ R. Chelakkot, R. G. Winkler, and G. Gompper, EPL 91, 14001 (2010).

${ }^{43}$ F. A. Messaud et al., Prog. Polym. Sci. 34, 351 (2009).

${ }^{44}$ S. K. R. Williams, J. R. Runyon, and A. A. Ashames, Anal. Chem. 83, 634 (2011).

${ }^{45}$ T. Shendruk and G. Slater, J. Chromatogr. A 1233, 100 (2012).

${ }^{46}$ J. Byrom, P. Han, M. Savory, and S. L. Biswal, Langmuir 30, 9045 (2014).

${ }^{47}$ A. Malevanets and R. Kapral, J. Chem. Phys. 110, 8605 (1999).

${ }^{48}$ J. T. Padding and A. A. Louis, Phys. Rev. E 74, 031402 (2006).

${ }^{49}$ C.-C. Huang, R. G. Winkler, G. Sutmann, and G. Gompper, Macromolecules 43, 10107 (2010).

${ }^{50}$ D. A. Fedosov, J. Fornleitner, and G. Gompper, Phys. Rev. Lett. 108, 028104 (2012).

${ }^{51}$ S. B. Babu and H. Stark, New J. Phys. 14, 085012 (2012).

${ }^{52} \mathrm{G}$. Gompper, T. Ihle, D. Kroll, and R. Winkler, "Multi-particle collision dynamics: A particle-based mesoscale simulation approach to the hydrodynamics of complex fluids," in Advanced Computer Simulation Approaches for Soft Matter Sciences III, edited by C. Holm and K. Kremer (Springer Berlin, Heidelberg, 2009), Vol. 221, pp. 1-87.

${ }^{53}$ E. Allahyarov and G. Gompper, Phys. Rev. E 66, 036702 (2002).

${ }^{54}$ T. Ihle and D. Kroll, Phys. Rev. E 63, 8321 (2001).

${ }^{55}$ K. Mussawisade, M. Ripoll, R. G. Winkler, and G. Gompper, J. Chem. Phys. 123, 144905 (2005).

${ }^{56}$ A. Malevanets and J. M. Yeomans, Europhys. Lett. 52, 231 (2000).

${ }^{57}$ M. Ripoll, K. Mussawisade, R. G. Winkler, and G. Gompper, Phys. Rev. E 72, 016701 (2005).

${ }^{58}$ E. Tüzel, T. Ihle, and D. M. Kroll, Phys. Rev. E 74, 056702 (2006).

${ }^{59}$ C. Huang, A. Chatterji, G. Sutmann, G. Gompper, and R. Winkler, J. Comput. Phys. 229, 168 (2010).

${ }^{60}$ A. Arnold et al., "Espresso 3.1: Molecular dynamics software for coarsegrained models," in Meshfree Methods for Partial Differential Equations VI (Springer, 2013), pp. 1-23.

${ }^{61}$ H.-J. Limbach, A. Arnold, B. A. Mann, and C. Holm, Comput. Phys. Commun. 174, 704 (2006).

${ }^{62}$ C.-C. Huang, G. Gompper, and R. G. Winkler, Non-equilibrium properties of semidilute polymer solutions under shear flow, J. Phys.: Conf. Ser. 392, 012003 (2012).

${ }^{63}$ D. Di Carlo, Lab Chip 9, 3038 (2009).

${ }^{64}$ L. E. Becker and M. J. Shelley, Phys. Rev. Lett. 87, 198301 (2001).

${ }^{65}$ S. Reddig and H. Stark, J. Chem. Phys. 135, 165101 (2011). 